\title{
EXTENSIVE CEREBRAL CALCIFICATION IN HYPOPARATHYROIDISM
}

Aditya Prakash Misraํㅜ, Ritu Mishra², Amit Gupta ${ }^{3}$

\section{HOW TO CITE THIS ARTICLE:}

Aditya Prakash Misra, Ritu Mishra, Amit Gupta. "Extensive Cerebral Calcification in Hypoparathyroidism". Journal of Evolution of Medical and Dental Sciences 2014; Vol. 3, Issue 37, August 21; Page: 9589-9593, DOI: $10.14260 /$ jemds/2014/3241

ABSTRACT: Extensive cerebral calcification with neuro-psychiatric symptoms is rare presentation of hypoparathyroidism. It is important to rule out other causes of intracerebral calcification by evaluation of hematological and Serum biochemical parameters. We report a case of 52 year old woman who presented with neuro-psychiatric symptoms, 20 years after a thyroid surgery. Marked improvement seen with calcium and vitamin D supplementation.

KEYWORDS: Extensive symmetric intra-cerebral calcification, hypo-parathyroidism, thyroid surgery.

INTRODUCTION: Physiological intracranial calcification is mostly asymptomatic and detected incidentally by neuroimaging. Pathological calcification is due to various causes, such as: metabolic disorders, infectious and genetic diseases. Hypoparathyroidism and pseudohypoparathyroidism are the most common causes of pathological calcification.

Intracerebral calcification in hypoparathyroidism is rare entity. We report a case of post thyroidectomy patient who presented to neurology OPD with neuro-psychiatric symptoms and extensive cerebral calcification was present on axial brain CT images. In this case importance of hormonal and mineral level evaluation in patients with neurological symptoms especially after thyroid surgery is emphasized. This disease has a good prognosis if detected early and treated.

CASE: A 52 year old woman presented with progressive dementia, behavioral disorders like psychosis, agitation, insomnia, nervousness, impaired concentration and memory impairment.she also had history of difficulty in walking, muscular cramps and intermittent tremor of the upper limbs during rest and activity. She had surgical history of thyroidectomy 20 years back.

On neurological examination, deep tendon reflexes (DTR) were exaggerated bilaterally; Babinski sign and palmo-mental reflex were present bilaterally. Slight extrapyramidal rigidity was present. Reaction towards painful stimuli was normal. Light touch was preserved. She recognized objects placed in the hand with closed eyes. Joint position and vibratory sensation was normal in the lower limbs.

CT scan revealed multiple symmetric intracerebral calcifications in both hemispheres (striatum, thalamus, periventricular white matter, centrum semiovale, corona radiata, and subcortical white matter) and in cerebellum (in dentate nucleus). Fine linear strips of calcification seen in corona radiata points towards perivascular nature of calcification.

Differential diagnosis included Physiological intracranial calcification and Pathological basal ganglia calcification is due to various causes as hypoparathyroidism (Post-thyroidectomy), pseudohypoparathyroidism, secondary hyperparathyroidism (in renal disease), Fahr syndrome.

Ultrasound of the abdomen revealed normal sized kidneys with no parenchymal lesions. Hematological investigations were normal. Keeping in mind her history of thyroidectomy she was additionally investigated for serum calcium, serum phosphorus and parathormone level. Serum 
biochemical investigation were serum calcium was $7.19 \mathrm{mg} / \mathrm{dL}$ (hypocalcemia), serum phosphorus level was $5.14 \mathrm{mg} / \mathrm{dL}$ (hyperphosphatemia), and parathyroid hormone level was low i.e. $<1.0 \mathrm{pg} / \mathrm{mL}$.

Patient was treated with calcium (1000 mg/day) and vitamin D (800 IU/day) for period of three months. General state of the patient improved. Symptomatic improvement was also appreciable. Frequency of upper limb tremors was reduced. Serum calcium level was $8.01 \mathrm{mg} \%$ on discharge after 4 weeks.

DISCUSSION: Patient had important psychiatric features, frequently described in hypoparathyroidism Psychiatric features are: anxiety, emotional lability, depression, confusion, hallucination, psychosis. ${ }^{1-7}$

Nervousness, impaired concentration and memory disturbance were initially thought of as a consequence of hypocalcemia. The clinical picture which points towards parathormone (PTH) deficiency usually consist of signs of spasmofilia (muscular cramps, Cvosteck and Trousseau signs, paresthesia, hyperexcitability, fatigue, anxiety). ${ }^{1-4}$

The following neurological signs may also be present: extrapyramidal signs (coreoathetosis, parkinsonian syndrome, dystonia, hemibalismus, and oculogiric crisis), epilepsy, spastic paraparesis, ataxia, dysarthria, dysphagia, papillary edema. Psychiatric features are: anxiety, emotional lability, depression, confusion, hallucination, psychosis.4,5

Babinski sign and palmomental reflex and slight extrapyramidal rigidity was seen in our patient.

The mechanism of intracerebral calcification in hypoparathyroidism is not completely known. Hyaline degeneration, calcifications in media and adventitia of the arterioles have been described. ${ }^{5}$

The phenomenon looks like being directly linked to abnormal serum levels of calcium and phosphate.

Differential diagnosis included Physiological intracranial calcification and Pathological basal ganglia calcification is due to various causes as hypoparathyroidism (Post-thyroidectomy), pseudohypoparathyroidism, secondary hyperparathyroidism (in renal disease), Fahr syndrome.

Hypoparathyroidism is an endocrine disorder caused as a result of congenital disorders, iatrogenic causes, infiltration of the parathyroid glands, suppression of parathyroid function, or idiopathic mechanisms. In these cases either there will be apparent deficiency of PTH secretion or end-organ failure. Idiopathic hypoparathyroidism is an uncommon condition of unknown etiology.

Acquired and congenital hypoparathyroidism would have either normal or undetectable PTH levels with hypocalcaemia whereas in idiopathic hypoparathyroidism both calcium and PTH levels are low. In pseudo hypoparathyroidism PTH levels will be high with hypocalcaemia.

In pseudo-pseudo hypoparathyroidism with increased PTH level both serum calcium and phosphorus levels are normal.

This patient was not having seizure, although seizures were reported as one of the most frequent cause of admission in a neurological department. ${ }^{1-5,8-10}$

Calcium administration had a good effect in neurological improvement. Clinical signs were markedly improved in our case by normalization of calcium level, as it was reported in other studies. ${ }^{1-5,11}$ 
Since adequate treatment of hypoparathyroidism may lead to marked clinical improvement, determination of serum calcium, phosphorus, and parathyroid hormone is mandatory in all individuals with calcification of the basal ganglia to rule out hypoparathyroidism.

\section{REFERENCES:}

1. Goltzman D, Cole DEC. Hypoparathyroidism. In: Favus MJ, ed. Primer on the Metabolic Bone Diseases and Disorders of Mineral Metabolism. Philadelphia, Pa: Lippincott-Raven; 1996: 220-3.

2. Cole DEC, Hendy GN. Hypoparathyroidism and pseudohypoparathyroidism. Endotext.com. 2005 http://www.endotext.org/parathyroid/ parathyroid9/parathyroidframe9.htm

3. Marx SJ. Hyperparathyroid and hypoparathyroid disorders. N Engl J Med. 2000; 343 (25): 186375.

4. Gonzalez-Campoy JM. Hypoparathyroidism. Updated: Jul 17, 2009 eMedicine Endocrinology. htm, http://emedicine.medscape.com/ article/122207-overview.

5. M Hossain. Neurological and psychiatric manifestations in idiopathic hypoparathyroidism: response to treatment. J Neurol Neurosurg Psychiatry 1970; 33 (2): 153-156.

6. Loeb JA, Sohrab SA, Hug M, Fuerst DR. Brain calcification induce neurological dysfunction than can be reversed by a bone drug. J Neurol Sci 2006; 243 (1-2): 77-81.

7. Kowdley KV, Coull BM, Orwoll ES. Cognitive Impairment and Intracranial Calcification in Chronic Hypoparathyroidism. The American Journal of the Medical Sciences: 1999; 317 (5): 273-277.

8. Bilge I, Sadýkoðlu B, Emre S, Irin A, Tatlý B. Brain calcification due to secondary hyperparathyroidism in a child with chronic renal failure. The Turkish Journal of Pediatrics 2005; 47: 287-290.

9. Fine A, Zacharias J. Calciphylaxis is usually non-ulcerating: Risk factors, outcome and therapy. Kidney International (2002) 61, 2210-2217.

10. Li ZJ, Huen W. Calciphylaxis with Arterial Calcification. NEJM 2007; 357(13):1327.

11. Goswami R, Goel S, Tomar N, et al. Prevalence of clinical remission in patients with sporadic idiopathic hypoparathyroidism. Clin Endocrinol (Oxf). Jun 222009.

Figure 1: Axial Ct images show symmetrical hyperdense calcification in bilateral corona radiate. Fine linear strips of calcification seen in corona radiata may suggests towards peri-vascular nature of calcification.

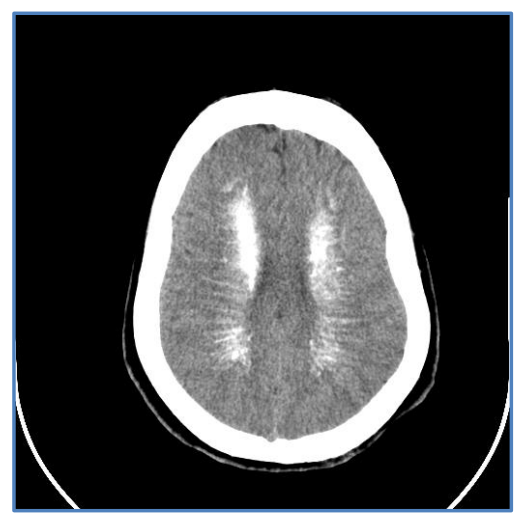

Fig. 1 
Figure 2: Dense symmetrical calcification of bilateral caudate and lentiform nucleus. Calcification also seen in bilateral thalamus.

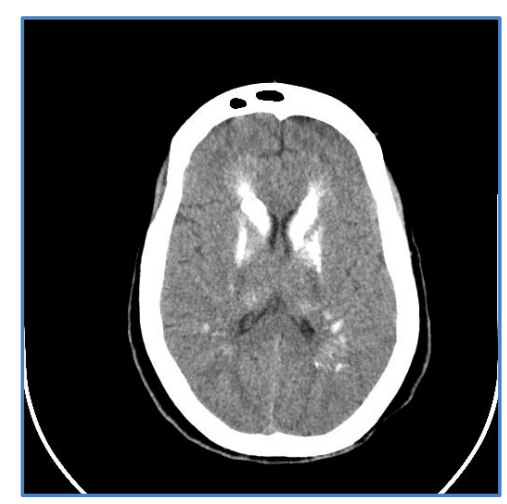

\section{Fig. 2}

Figure 3: Axial Ct images showing subcortical white matter calcification in bilateral parietooccipital region. In addition to bilateral basal ganglia calcification calcification of internal capsule also noticed.

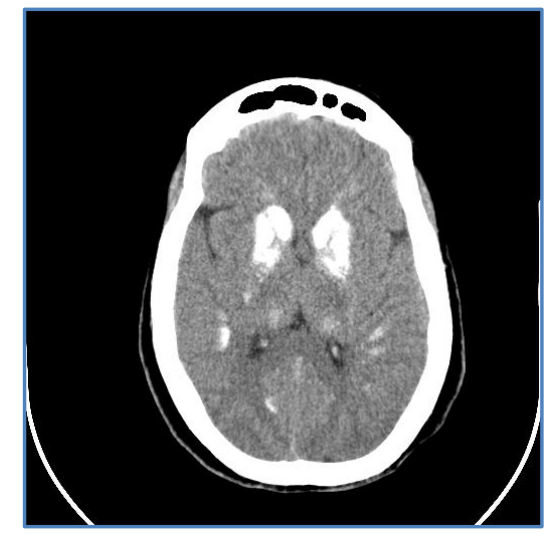

Fig. 3

Figure 4: Hyperdense symmetrical calcification of dentate nucleus seen on axial CT images.

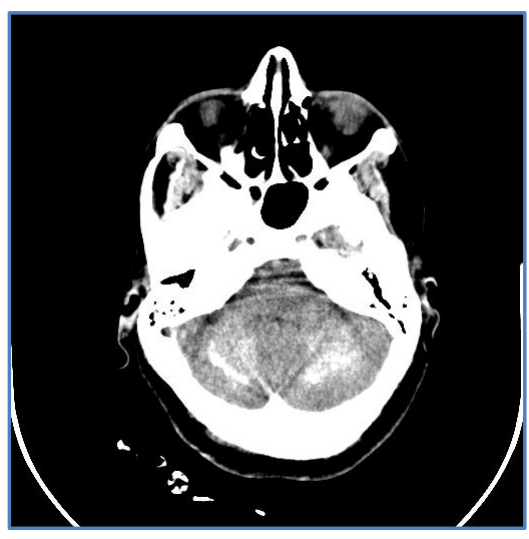

Fig. 4 


\section{CASE REPORT}

\section{AUTHORS:}

1. Aditya Prakash Misra

2. Ritu Mishra

3. Amit Gupta

\section{PARTICULARS OF CONTRIBUTORS:}

1. Associate Professor, Department of Radiodiagnosis, Rama Medical College, Hospital and Research Centre, Kanpur.

2. Assistant Professor, Department of Obstetrics and Gynaecology, Rama Medical College, Hospital and Research Centre, Kanpur.

3. Assistant Professor, Department of Neurology, Rama Medical College, Hospital and Research Centre, Kanpur.

\section{NAME ADDRESS EMAIL ID OF THE} CORRESPONDING AUTHOR:

Dr. Aditya Prakash Misra, $2 \mathrm{a} / 43$,

Azad Nagar,

Kanpur-208002.

Email: saggimishra@rediffmail.com

Date of Submission: 28/07/2014.

Date of Peer Review: 30/07/2014.

Date of Acceptance: 14/08/2014.

Date of Publishing: 19/08/2014. 